\title{
Modeling Uncertainty and the Economics of Climate Change: Recommendations for Robust Energy Policy
}

\author{
A. Haurie • M. Tavoni • B. C. C. van der Zwaan
}

Published online: 15 July 2011

(C) Springer Science+Business Media B.V. 2011

Keywords Modeling · Uncertainty . Climate change . Energy policy

Economic analysis of climate change has become a fundamental instrument for the multidisciplinary research of this global environmental challenge and is increasingly used for informing the ongoing discussion between climate scientists and policy makers. Much progress has been achieved in climate change economics over the past decade, which has led to the refinement of "integrated assessment models" and the development of other approaches that permit analyses of the multiple dimensions of climate change, either individually or jointly. Of particular relevance

\author{
A. Haurie \\ ORDECSYS, \\ Geneva, Switzerland \\ M. Tavoni \\ Princeton University, \\ Princeton, NJ, USA \\ M. Tavoni \\ Fondazione Eni Enrico Mattei, \\ Milan, Italy \\ B. C. C. van der Zwaan $(\bowtie)$ \\ Policy Studies Department, Energy Research Centre of the \\ Netherlands (ECN), \\ Amsterdam, The Netherlands \\ e-mail: vanderzwaan@ecn.nl \\ B. C. C. van der Zwaan \\ Lenfest Center for Sustainable Energy, Earth Institute, \\ Columbia University, \\ New York, NY, USA \\ B. C. C. van der Zwaan \\ School of Advanced International Studies, \\ Johns Hopkins University, \\ Bologna, Italy
}

in this context are cost-benefit and cost-effectiveness analyses. The formulation of climate policy is increasingly becoming reliant on the adequacy of economic analysis, yet many of its aspects are left poorly understood. Both the scientific and policy-making communities therefore agree that economic studies of climate change ought to be perfected.

Among the subjects that deserve further in-depth investigation, the issue of uncertainty emerges as, perhaps, the most prominent. The outpouring of literature following the publication of the "Stern Review of the Economics of Climate Change" has brought fundamentally new insights to the field of climate change research, in particular, with regards to the uncertainties by which climate change is intrinsically characterized [2]. Although a number of numerical economic analyses of climate change have employed techniques that in some form account for uncertainty, such as with Monte Carlo simulations or through stochastic differential equations [4], it has become clear that more advanced techniques are needed to more suitably design and evaluate greenhouse gas mitigation policies [3].

Scientists have recently extensively explored the model and parameter space of the economics of climate change, and especially the Stern Review has catalyzed a fundamental rethinking of the economic rationale for action on global warming [1]. While the conditions sufficient to provide a case for strong $\mathrm{CO}_{2}$ abatement activity can today be readily formulated, much more work is needed to create a satisfactory account of the relevant economics. In particular, it needs to be better understood how climate policy makers should handle the abundance of natural and social scientific uncertainties in the field of climate change.

This special issue of Environmental Modeling and Assessment is meant to gather front-edge research and innovative analysis in the modeling of uncertainty related to the economics of climate change. The focus is notably on advancements in probabilistic integrated assessment modeling 
and stochastic analysis of climate futures. The possibility to use non-probabilistic economic methods to treat uncertainty in global or regional dynamic climate change models is explored as well. Given the intimate link between climate change and the nature of mankind's energy production and consumption system, this special issue also proffers direct practical recommendations for energy decision making at the global, regional, and national levels.

The special issue originated from a series of research tasks carried out under the PLANETS project, funded by the European Commission under its $7^{\text {th }}$ Framework Programme and co-coordinated by the Fondazione Eni Enrico Mattei (FEEM) and the Energy research Centre of the Netherlands (ECN). ${ }^{1}$ This project, accomplished in 2010, had, as main focus, how to incorporate uncertainty when carrying out numerical analysis of climate and energy policies. A special PLANETS session was organized during the 2010 edition of the International Energy Workshop (IEW 2010, Royal Institute of Technology, Stockholm), which generated broad expert discussion on both methodology and policy-related issues. The recognition of the importance of these topics and the diversity of approaches undertaken, plus a concern over them becoming fragmented in the literature, constituted the motivation to edit this special issue gathering the generated material in one orchestrated publication.

Several contributions, in the form of 12 papers, have been brought together with the aim of providing a comprehensive overview of some of the main recent developments in the modeling of uncertainty in the economics of climate change. We categorize these 12 articles in five distinct domains in hybrid integrated assessment EEE (Energy-EnvironmentEconomy) modeling:

- Robust optimization and viability theory:

1. Dynamical allocation method of emission rights of pollutants by viability constraints under tychastic uncertainty;

2. Energy security: a robust optimization approach to design a robust European energy supply via TIAM-WORLD;

- Real options analysis and robust energy technology portfolios:

3. Robust energy portfolios under climate policy and socio-economic uncertainty;

- Statistical analysis through Monte Carlo simulations and stochastic optimization:

4. Combining stochastic optimization and Monte Carlo simulation to deal with uncertainties in climate policy assessment;

\footnotetext{
${ }^{1}$ See www.feem-project.net/planets/.
}

5. Statistical simulation to estimate uncertain behavioural parameters of Hybrid Energy-Economy Models;

6. Using an allowance reserve to manage uncertain costs in cap-and-trade programs for greenhouse gases;

- Value of information internalization:

7. The value of better information on technology $\mathrm{R} \& \mathrm{D}$ programs in response to climate change;

- Stochastic programming:

8. Mitigation portfolio and policy instruments when hedging against climate policy and technology uncertainty;

9. Evaluating uncertain $\mathrm{CO}_{2}$ abatement over the very long-term;

10. The benefits of cooperation under uncertainty: the case of climate change;

11. Anticipating climate threshold damages;

12. The impact of uncertainty in forcing targets and $\mathrm{CO}_{2}$ storage availability on long-term climate change mitigation.

In what follows, we first provide a brief overview of some of the most interesting insights obtained in these respective studies. We next draw a few overall conclusions and recommendations that derive from the ensemble of these 12 articles.

\section{Overview of the Main Insights}

In Dynamical Allocation Method of Emission Rights of Pollutants by Viability Constraints under Tychastic Uncertainty, Aubin, Chen, and Durand deal with the intricate issue of how to translate an overall climate mitigation objective into an allocation of emission reduction objectives among polluters. The study proposes a method for dynamically allocating pollutant emissions rights among polluters, given that the emissions growth rates of the various polluters cannot be controlled, or even predicted. The problem is solved with mathematical and algorithmic tools of viability theory. Knowing the maximum growth rates of emissions of each polluter in the worst case, the method of these authors provides the allocation rule for emissions rights and the required initial emissions. It assures that, whatever the growth rates of the emissions below the maximum growth rates, the resulting emissions will be, both globally and locally, under their thresholds.

Robust optimization is used to evaluate the topic of energy security in Energy security: a robust optimization approach to design a robust European energy supply via TIAM-WORLD, by Babonneau, Kanudia, Labriet, Loulou, and Vial. Several sources of randomness motivate the need 
for incorporating uncertainty when dealing with energy security, as partial or total closure of energy supply routes can occur because of technical, economic, or political reasons, all highly susceptible to risk. The paper applies the robust optimization method to a large-scale EnergyEconomy model (TIAM-WORLD) by making minimalist assumptions on probability distributions but ensuring a high level of reliability. Results indicate that supply of energy can be guaranteed with a known probability, under the mild assumption that the mean of the random availability factors be known, or bounded at some level. Moreover, the authors show that such reliability is achieved at what may be considered a moderate extra cost, not exceeding $0.7 \%$ of total EU energy costs, with the additional benefit of significantly reducing the concentration of supply sources.

In Robust Energy Portfolios under Climate Policy and Socio-economic Uncertainty, Szolgayová, Fuss, Khabarov, and Obersteiner analyze the choice of technology portfolio needed to achieve robust long-term climate risk mitigation. The paper assesses optimal technology portfolios across different socio-economic scenarios for a range of stabilization targets, using a two-stage process in which the dynamics underlying technology adoption and operational decisions are analyzed in a real options model, the output of which feeds into a larger optimization model. The analysis has enabled to gain insights into much debated biomass mitigation options, showing that incorporating uncertainty about technological developments and the timing of commercialization is likely to be a considerable barrier to the contribution of biomass in the optimal energy portfolio. Finally, mitigation strategies of a risk-averse investor are shown to be very sensitive to the uncertainty surrounding climate goals, which gives an indication for policymakers about the importance of clearly communicated commitments and credibility.

A broad assessment of the impact of uncertainty for climate policy is carried out in Combining Stochastic Optimization and Monte Carlo Simulation to Deal with Uncertainties in Climate Policy Assessment by Babonneau, Haurie, Loulou, and Vielle. The authors use stochastic programming in a bottom-up EEE model and Monte Carlo simulation in a top-down one, combined with ex post statistical analysis, to evaluate uncertainties related to climate, technology, economy, and energy prices. Their results have highlighted the fundamental role of uncertainty over climate sensitivity, which has a major impact on the attainability and costs of climate policies. Concerning the technological aspects of climate policy, the availability and composition of carbon-free technologies have also shown to be determinant. The paper has also shown that other factors - in particular, the price of oil and the behavior of OPEC - are likely to affect the success and the cost of climate policy.
In Statistical Simulation to Estimate Uncertain Behavioural Parameters of Hybrid Energy-Economy Models, Beugin and Jaccard address one of the fundamental issues in integrated assessment models, namely that of the estimation and calibration of the input parameters. This is especially important when dealing with behavioral parameters that portray firm and household technology choices. These are characterized by a large number of uncertainties and are affected by a lack of historical data on technology capital costs, operating costs, and market shares. The authors tackle these issues by applying Bayesian statistical simulation for estimating the most likely values for these key behavioral parameters, in order to best explain past technology choices. Markov-Chain Monte Carlo is used to estimate the posterior probability distribution functions of the parameters, from which the authors then sample to simulate policies to influence future technology choices. The method has highlighted several challenges related to calibrating and simulating EEE models. Computational issues, data limitations, and structural modeling uncertainty pose significant constraints to the applicability of established methodologies to these models. Furthermore, forecasts generated by the sampling from the parameter distribution were fairly robust to the estimated uncertainty over the behavioral parameters.

Monte Carlo techniques are also used in Using an allowance reserve to manage uncertain costs in cap-andtrade programs for greenhouse gases, though for a different purpose. Golub and Keohane investigate the design and performance of a $\mathrm{CO}_{2}$ allowance reserve in the context of a cap-and-trade program and assess the capacity of the allowance reserve that smoothens out the response of allowance prices to significant but temporary shocks. The paper uses a Monte Carlo approach in which the parameters of the marginal abatement cost function and the supply of offsets are drawn from specified distributions. Their results suggest that an allowance reserve similar to recent proposals for US climate legislation can be effective in containing allowance prices. The model is used to explore the trade-off among the most policy sensitive features of the allowance reserve: its total size, the trigger price, and the degree of confidence that the reserve will be large enough to limit allowance prices to the target level. Their result indicates that a lower trigger price, or a higher degree of confidence, would require a larger reserve.

Baker and Peng address the topic of how to include uncertainty in the analysis of climate change policy and especially the evaluation of R\&D policy, in The value of better information on technology $R \& D$ programs in response to climate change. The authors' aim is to calculate the economic value of including expert elicitations, a new method for determining how R\&D investments may achieve technological advance, though a quite time- 
consuming and resource-intensive one. By using baseline elicitations from one study, the paper generates estimates for the Expected Value of Better Information (EVBI) by revisiting and improving earlier assessments. The results range from $\$ 100$ million to $\$ 11$ billion for individual technology categories - thus indicating that the EVBI is many orders of magnitude larger than the cost of collecting such information, particularly so for technologies that require large $R \& D$ investments. This indicates that it may be worthwhile to move forward with large-scale, carefully managed elicitations on climate-friendly energy technology.

In Mitigation portfolio and policy instruments when hedging against climate policy and technology uncertainty, De Cian and Tavoni study how policy and technology uncertainty affect the incentive to invest differently in the portfolio of climate change mitigation options. The authors use a stochastic integrated assessment model to evaluate the effects of uncertainty about future carbon taxes and costs of low carbon power technologies and assess the potential for regulation on emissions performance and renewable portfolio in accompanying a market-based climate policy. Results suggest that carbon price uncertainty does not substantially change the level of abatement, but it does have an influence on the composition of the mitigation portfolio, for example, by reducing $R \& D$ investments in clean energy technologies. On the basis of these results, the authors provide some guidance on how to support specific regulation to incentivize investments in CCS and clean energy R\&D, as a complementary policy to carbon pricing.

Gerlagh and van der Zwaan, in Evaluating Uncertain $\mathrm{CO}_{2}$ Abatement over the Very Long-Term, also apply stochastic programming to an integrated assessment model to evaluate the effectiveness of CCS as a climate mitigation option, when the very long-term (e.g., until the year 3000) is taken into account. The authors model uncertainty regarding climate change damages and leakage from a $\mathrm{CO}_{2}$ reservoir and find that $\mathrm{CO}_{2}$ leakage does eventually not reduce the effectiveness of CCS very much when one assumes a descriptive (high) value for the discount rate, even in combination with high climate change damage assumptions. On the contrary, with a prescriptive (low) value for the discount rate, leakage is shown to become problematic: leakage with a mean residence time of 100 years reduces the attractiveness of CCS substantially. The paper shows that uncertainty regarding the value of the leakage rate and the extent of climate-induced damages to the global economy should not prevent us from deploying CCS on a large scale, but that CCS ought not to be fully exempt from carbon taxes.

In The benefits of cooperation under uncertainty: the case of climate change, Brechet, Thenie, Zeimes, and Zuber analyze how taking uncertainty into account in an integrated assessment model impacts policy design and incentives to cooperate for different countries. Using a stochastic model with uncertainty over climate sensitivity, the authors show that the degree of convexity of the damage function plays a crucial role in determining in which direction uncertainty changes policy recommendations, especially if one considers the diversity of impacts at the country and regional level. Thus, more empirical research is warranted on estimating regional damage functions. Finally, the paper emphasizes that, when uncertainty is taken into account, new reasons to cooperate appear, specifically the reduction in risks of climate damages.

The uncertainty over climate sensitivity and climate damages is also assessed in Anticipating Climate Threshold Damages, by Lorenz, Schmidt, Kriegler, and Held. The authors use an integrated assessment model to calculate the welfare benefits from resolving the uncertainty and to analyze the implications of anticipation of future learning for optimal near-term climate control. The results indicate that the welfare benefits from learning were significant, but benefits due to the anticipation of learning were not, thus confirming previous results in the literature. If learning about an irreversible threshold is included, though, the authors show that anticipation can become crucial both in terms of necessary adjustments of pre-learning emissions and resulting welfare gains.

In The impact of uncertainty in forcing targets and $\mathrm{CO}_{2}$ storage availability on long-term climate change mitigation, Keppo and van der Zwaan use a stochastic version of a bottom-up energy systems model to study the impacts that uncertainty about the CCS storage potential and climate control target may have on transitional climate mitigation strategies. The authors find that, if a stringent climate target is a possibility, it tends to dominate the solution: If deep $\mathrm{CO}_{2}$ emission reductions are not started as soon as possible, the target may become unreachable. Regarding technological options, CCS is shown to remain an important option for mitigation, though when stringent climate policies are considered its use is higher in the mid-term but lower in the latter half of the century, when a fully carbon-free energy system is required.

\section{Overall Conclusions and Recommendations}

Notwithstanding the variety of approaches and topics tackled in this collection, several key outcomes regarding the modeling of uncertainty in climate change economics appear robust, and thereby a couple of recommendations emerge. We summarize them in three main points:

- Uncertainty over the performance and acceptability of clean energy technologies and over the policies designed to stimulate their implementation exerts a profound impact on the nature of climate mitigation 
strategies. In particular, technology and policy uncertainties affect the breakdown of mitigation portfolios (De Cian and Tavoni), as well as the deployment of innovative mitigation options (Szolgayová et al.). More specifically, they influence the diffusion of key alternatives to fossil fuels such as CCS (Gerlagh and van der Zwaan, Keppo, and van der Zwaan) and have an effect on the scope for changing firm and household behavior (Beugin and Jaccard). A better understanding of the technological features of these climate mitigation options and of their implementation prospects and opportunities for improvement constitutes a top research priority, for which tools like expert elicitation may offer a promising avenue (Baker and Peng).

- Incorporating uncertainty into EEE models of climate change can yield important insights regarding how to deal with unresolved issues such as burden sharing (Aubin et al.), how to ascertain security of energy supply (Babonneau et al.), and how to design effective climate policy instruments (Keohane and Golub). Tools like the ones discussed in this special issue may advance our understanding of energy and environmental challenges, and their application may contribute to their solution.

- Fundamental uncertainties in the climate sciences, such as on the value of the climate sensitivity and the monetization of the large variety of climate damages, remain of utmost importance for determining the attainability and costs of climate policies (Babonneau et al.). They also affect the establishment of incentives for international cooperation on climate control (Brechet et al.) and the realization of near-term climate management strategies (Lorenz et al.). More dialog and collaboration between climate and social scientists could allow improving the modeling and understanding of uncertainty in this field.

The 12 papers constituting this special issue provide a wide array of both modeling and policy instruments. These tools address a broad variety of different problems and analyze them with a great diversity of distinct model types. The latter range from purely analytical methods to medium-size and large-scale numerical models. This collection of articles has shown that, for each class of problems, specific ways exist to handle uncertainty - ways that, on the one hand, do not compromise the setup of the model, while on the other hand not yielding computational infeasibilities or numerical constraints. Most of the proposed approaches can be further extended and improved, by which they could provide more definitive answers regarding the role of uncertainty in the field of climate change economics. To this end, multidisciplinary cooperation is even more warranted than exercised to date, in order to bridge the knowledge gap that the community dedicated to this research area is still observing. Such collaboration is also essential to refine its ability to communicate complex uncertainties to a broader non-technical audience. In our view, increasingly relevant bodies such as the Intergovernmental Panel on Climate Change (IPCC) offer the unique opportunity to bring together different sorts of expertise and skills. We hope this special issue may, perhaps, instruct the Working Group III authors of the fifth Assessment Report (AR5) of the IPCC in their efforts to publish a newly updated overview of climate mitigation sciences in 2014.

\section{References}

1. Heal, G. (2009). The economics of climate change: A post-stern perspective. Climatic Change, 96, 275-297.

2. Stern, N., et al. (2006). The Economics of Climate Change: The Stern Review. Cambridge, UK: Cambridge University Press.

3. Weitzman, M. (2009). On modeling and interpreting the economics of catastrophic climate change. Review Economics Statistics, 91(1), $1-19$.

4. Zapert, R., Gaertner, P. S., \& Filar, J. A. (1998). Uncertainty propagation within an integrated model of climate change. Energy Econ, 20, 571-598. 